\title{
Mathematical Modeling of Thrombus Growth
}

\section{Phenomenological Models}

\author{
A. Kondratyev \\ Department of Physics, Herzen State Pedagogical University of Russia, \\ Moika River Embankment 48, St. Petersburg 191168, Russia

\section{A. Lyaptsev} \\ Department of Physics, Herzen State Pedagogical University of Russia, \\ Moika River Embankment 48, St. Petersburg 191168, Russia
}

\section{Mikhailova}

Institute of Experimental Medicine, Federal Almazov Medical Research Centre, Akkuratova str.2, St.Petersburg 197341, Russia

Copyright (C) 2014 A. Kondratyev, A. Lyaptsev and I. Mikhailova. This is an open access article distributed under the Creative Commons Attribution License, which permits unrestricted use, distribution, and reproduction in any medium, provided the original work is properly cited.

\begin{abstract}
Phenomenological mathematical model of laser-induced thrombi growth is developed on the basis of Bogolubov's hierarchy of time scales. The stochastic character of thrombi growth is revealed in the model by explicit introduction of the probability function. The main foundations of the model correspond to the basic experimental results concerning thrombus formation obtained in recent years. The modeling curves permit to achieve qualitative agreement between model and experimental data. The comparison of the model with other models of thrombus growth is performed.
\end{abstract}

Mathematics Subject Classification: $92 \mathrm{C} 05$

Keywords: Mathematical model, microvessels, thrombus growth, platelets, hierarchy of time scales 


\section{Introduction}

The art of good mathematical modeling relies on (i) a sound understanding and appreciation of a biological problem; (ii) a successful mathematical representation of the biological phenomena; (iii) qualitative analysis of a mathematical model and finding useful solutions; and finally: (iv) a proper biological interpretation of the mathematical results in terms of insights and predictions [8]. As declared in [14], mathematical biology is the most exciting modern application of mathematics. Non-vanishing interest to the problem of mathematical modeling of thrombus growth in mesenteric blood vessels demonstrated in the last decade confirms this statement $[1,15,22,24-26,29,30]$. Theoretical investigations were stimulated by a set of new experimental results concerning thrombus formation obtained in recent years $[6,13,19,20,23,27]$.

These experimental studies demonstrate the complexity of the platelet aggregation process in vivo in flowing blood, when the majority of platelets tethering to the luminal surface of a developing thrombus subsequently translocate and detach, with only a small percentage of tethered platelets forming stationary adhesion contacts at both arterial and venous shear rates. In response to vascular and tissue trauma, platelets establish adhesive interactions with exposed subendothelial structures. The adhesion of platelets to the injured vascular wall or to the surface of a growing aggregate is a principally nonlocal process: a platelet adheres to the surface only if the distance from its center to the point of fixation is smaller or equal to its size.

Another important aspect of hemostasis is the near-wall blood layer concentration with platelets. Platelets are displaced from the core of the blood flow by erythrocytes migrating from the walls to the axis of the flow. The dispersion of platelets causes the dispersion of the surrounding plasma and the platelets contained in it. Platelet aggregation and coagulation may seem to be largely independent processes that only interact when the fibrin mesh forms on the platelet aggregate. But there are important interactions much earlier in the processes that couple them.

Many new experimental results were obtained concerning the process of platelet activation which can be induced by direct contact of platelets with collagens exposed in the injured vessel tissue, by the action of thrombin, or by other platelet-secreted plasma-phase chemical messengers like adenosine diphosphate and thromboxane- $\mathrm{A}_{2}$. Thrombin is the final enzyme produced by the coagulation cascade, and it plays several roles in coagulation: it promotes the formation of a fibrin mesh, and stabilizes mechanically the platelet aggregates that have formed on the injured vessel. Thrombin also promotes its own production by different activating factors and by activating platelets.

The main tendency revealed in the presented theoretical publications $[5,11,17$, 28 ] is an attempt to describe tiny details of the processes mentioned above and 
other interactions and to create a complete mathematical model of the phenomenon. This tendency leads to the extreme complexity of developed models, and either makes the model as a whole useless for a qualitative analysis and predictions or makes to proceed with significant simplifications resulting in the loss of the initially introduced details. We will briefly discuss two typical models of this kind offered in [11] and [26].

The mathematical model developed in [11] includes both plasma-phase and membrane-phase reactions that occur after the exposure in a thin boundary layer shell above the injured surface. The assumption that all species in this shell are well mixed leads to the possibility to describe all reactions by means of ordinary differential equations. Three different populations of platelets are considered in the model: (i) inactivated and free in solution, (ii) activated and attached directly to the subendothelium, and (iii) activated but not attached directly to it. Then a set of assumptions is made concerning the three ways of inactivated platelets to become activated, concerning the number of platelet layers that are allowed to form, etc. Special group of assumptions is devoted to the properties of reactants and to the binding of proteins to surfaces. As a result, the model contains 59 differential equations with even more numerous set of parameters that determine the factors entering the equations.

The model combines a fairly comprehensive description of coagulation biochemistry, interactions between platelets and coagulation proteins, explicit treatment of protein binding site densities, and effects of chemical and cellular transport. Thus, this model permits describing some separate features of the phenomenon but also it is sure to prompt the question of whether essentially any experimental observation could be fit by adjusting parameter values appropriately. The assumption of well-mixedness becomes suspect for large reaction regions because of the potential effects of upstream zymogen and platelet depletion, and the downstream effect of enzymes produced upstream. The model requires further development to allow for spatial heterogeneity, and its real value can be estimated only after the calculation or independent experimental determination of the values of all parameters entering the theory becomes possible.

The continuous mathematical model of platelet thrombus formation in blood flow developed in [26] is based on partial differential equations (PDE) and takes into account all basic processes, such as the transport of platelets along and across the blood flow, their adhesion to the injured part of a vascular wall and to the surface of growing thrombocyte aggregate, the activation, the emission of activators, and the aggregation of platelets. The model consists of a system of 10 linked PDE and involves the so called level set approach (LSA) to tracing the domains interface boundary motion. It allows finding the distance to the boundary by using the additional differential equation describing the boundary movement, and thus gives the ability to distribute the surface processes over the transition zone of a finite length. A network of vessels is described in this model by onedimensional flows of Poiseuille type in regular parts with parallel walls and twodimensional Navier-Stokes description in the zones of more complicated geometry - bifurcations and clot formation areas. 
The main difficulties in the description of the process of the thrombocyte growth into the blood flow occurring due to the adhesion of platelets carried by the flow is caused (like in all other models) by the principal nonlocality of the adhesion and by the necessity to calculate the rate of this process on the moving boundary of the aggregate. In order to trace the movement of the aggregate's boundary in the frame of LSA method the sharp boundary of the domain, where a jump of physical properties of the medium occurs, is replaced by the transition zone of their continuous variation. The blood is considered as a one-phase fluid with constant density and viscosity, neglecting the difference in the densities of erythrocytes and the plasma and non-Newton properties of blood.

The complexity of the model caused some simplification in the process of numerical calculations. In particular, only one the basic soluble activator of platelets (ADP) was taken into account. The results of the numerical calculations were used for a direct comparison with the results of a classical experimental work [2]. The comparison showed that the mathematical model [26] is a sufficiently complete model of hemostasis, but it should be clearly stated that the agreement with the experimental data in [2] can be obtained in the frame of much simpler phenomenological mathematical models $[1,8]$.

Despite considerable advantages of the discussed complex mathematical models which testify to the necessity of their further development, there are certain deficiencies that should be remedied. First of all, it should be mentioned that the number of considered elementary processes that determine the final picture of thrombus formation, is much larger in [11] than in [26]. Even in the cases when the same basic elementary microscopic processes were considered, different assumptions were involved. For example, the platelets, depending on their state, are divided in [11] into three groups, and in [26] - into four groups. Qualitative agreement with experimental results demonstrated by both models, testifies to a certain independence of this picture from the details of elementary interactions in the biological system.

The process of thrombus formation is a very complex phenomenon to taking into account the details which were neglected in the existing models and which can be added by future experimental investigations. Even relative importance of various elementary processes can be different. The complexity of the phenomenon also means that all the coefficients entering the equations are the sums of the contributions of different elementary processes leading to the same results in the final picture. Thus, the modeling of the phenomenon at present time can be produced in the frame of the Bogolubov's hierarchy of time scales, what was done in [1], but in implicit form: the hierarchy of time scales was taken into account not by the introduction of corresponding dimensionless parameters, but by the assumption that only the most "slow" process in the phenomenon must be traced by "dynamical" description, and "quick" processes can be taken into account in terms of stationary values of their parameters. The complexity of the model can be reduced to some extent when tracing the time scale hierarchy in explicit way.

The independence of the final picture of the tiny details means that all separate processes occurring in the phenomenon can be divided into "quick" and "slow" ones. 
This clearly demonstrates the existence of several stages of the process, so that mathematical models for different stages of the phenomenon can be different: time dependence of the evolution of slow variables would not depend on the details of the dynamics of quick variables. In this sense, rather simple phenomenological mathematical models offered in $[1,8,9,16]$ correspond to a rough time scale, and consequently, to a smooth and slowly varying in time picture of the phenomenon although it was not shown in the explicit way. These models turned to be in a good qualitative agreement with corresponding experimental data $[2,21]$. At the same time, certain restrictions for the allowed values of the entering parameters, peculiar to the models, can and should be removed (what is desirable in correspondence with the discussion about the values of the coefficients entering the equations) by a usage of different mathematical technique on the basis of the same biological and physical considerations.

This paper is devoted to the development of a new phenomenological model of laser-induced thrombosis on the basis of the analysis of the hierarchy of time scales. The model is developed as a generalization of the initial Richardson's approach to the problem [18].

\section{Phenomenological theory of thrombus formation}

Main directions of the generalization of the Richardson's phenomenological model presented in $[1,8]$ are: 1) the dependence of platelet activation time on the distance from the injured vessel wall; 2) the heterogeneity of the platelet distribution in blood flow in the vicinity of the vessel wall; 3) the adequate choice of the phenomenological function describing the dependence of blood velocity on the thrombus size. A detailed qualitative discussion of these directions can be found in [8]. Here we will only emphasize that these directions are in the complete agreement with the known experimental results $[4,7,10,13]$ and with the results of theoretical investigation $[11,26]$. Rather different directions of generalization of the Richardson's phenomenological model will be followed in this work and their mathematical realization also will be different.

The elaborated analysis of the biological factors and of the corresponding equations presented in the most detailed for the present time picture of thrombus formation [11] leads to the following conclusions: 1) different factors form the groups that perform the same influence on the process of thrombus formation being different only in the sense of their time scale; 2) the process of thrombus growth is the most "slow" process compared with the different "quick" processes determining the picture under consideration. As a result it can be considered that the time evolution of the "slow" process (the rate of thrombus growth) doesn't depend on the dynamics of "quick" processes. It depends only on a set of dimensionless parameters determined by the ratios of typical time lengths of "quick" and "slow" processes. 
We start a construction of a new mathematical model of thrombus formation based on the Bogolubov's hierarchy of time scales by brief comments concerning experimental curves on Fig. 1 which present the dependence of blood velocity on thrombus size [21].

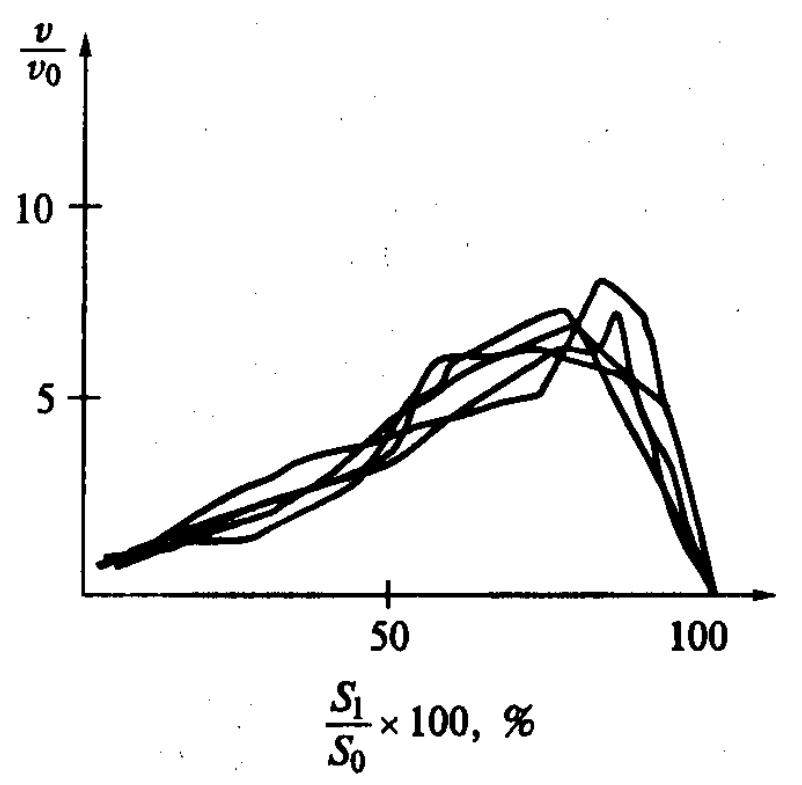

Fig.1

The concave and the convex parts on the curves corresponding to the experiments with different animals do not coincide. This testifies to the fact that the mutual relative role of different biological factors discussed above was different in these cases. The similar resulting behavior of the curves means that in the frame of the phenomenological model it is not necessary to consider those biological factors separately. The same comments can be made concerning the dots representing the blood velocities on Fig.2 [21].

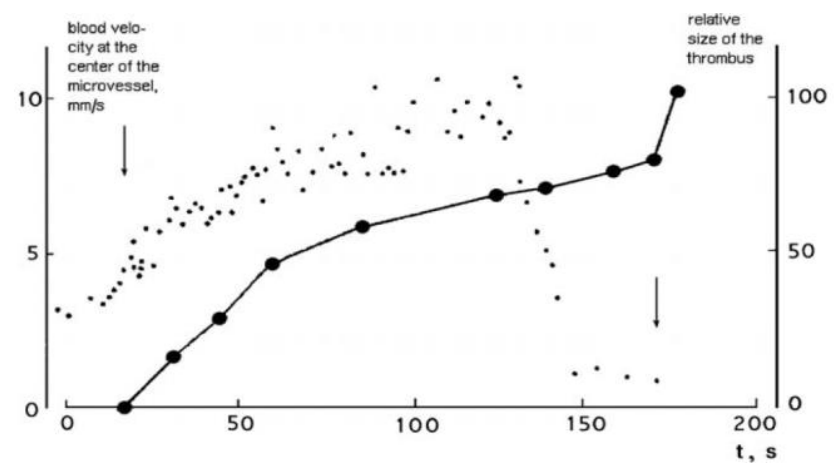

Fig.2. 
The introduction of a set of dimensionless parameters that rule the process of thrombus growth can be performed on the basis of a following model of blood flow in a mesenteric vessel (Fig.3).

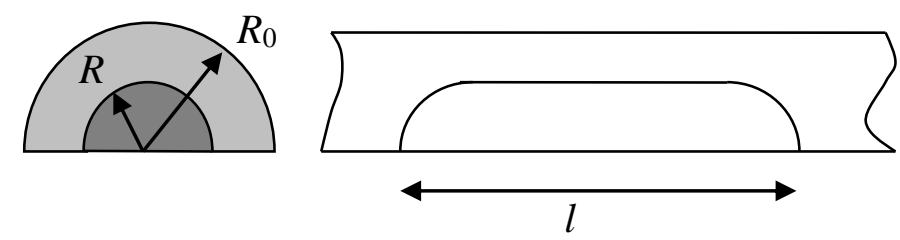

Fig.3.

We will consider a thrombus with a shape shown on the Fig.3 (the case of more complex shape can be considered in a similar way changing the rigid model for a soft one) and assume its length $l$ to be proportional to its radius $R: l=C_{1} R$, where $C_{1}$ is a dimensionless constant of the unit order. We will also assume that the activated platelets aggregating to the growing thrombus move on the distance $b$ from its surface (Fig.4).

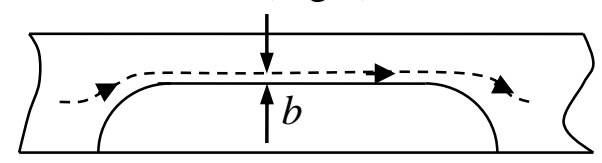

Fig.4.

The velocity $v$ of blood flow in the thin layer $b$ according to Richardson can be given by the expression [18]

$$
v=\Gamma b .
$$

Gradient of the velocity $\Gamma$ is proportional to the ratio of mean value of the velocity to the linear size of the cross section of the vessel, i.e. to $\left(R_{0}-R\right)$. Mean value of the velocity equals to the ratio of the blood flow rate $Q$ (volume of blood flowing past a given point per unit time) to the area of the cross section. Taking into account that $b<R_{0}-R$ we get the following expression for $v$ :

$$
v=\frac{C_{2} Q b}{\left(R_{0}-R+b\right)\left(R_{0}^{2}-R^{2}\right)} .
$$

Thus, the time $t$ a platelet needs to cover the distance $l$ equals to

$$
t_{p}=\frac{l}{v}=\frac{C_{3} R\left(R_{0}-R+b\right)\left(R_{0}^{2}-R^{2}\right)}{Q b} .
$$


This time must be of the same order of magnitude as the activation time $t_{A}=0.1 \div 0.2 \mathrm{c}$. what makes possible the aggregation of the platelet to the growing thrombus. This process has a stochastic character, so it is necessary to introduce a probability $w\left(t_{p}\right)$ of the platelet's attachment to the thrombus. The probability $w\left(t_{p}\right)$ can be approximated by some smooth function which is close to unity when $t_{p}>t_{\max }$ and close to zero when $t_{p}<t_{\min }$, where $t_{\max }$ and $t_{\min }$ are associated with the activation time $t_{A}$ and present another way of introduction its dependence on the distance than used in [11]. For example, such a function can be defined in terms of the hyperbolic tangent function:

$$
w\left(t_{p}\right)=w_{0}+\frac{1}{2}\left(1+w_{0}+\left(1-w_{0}\right) \operatorname{th}\left(\alpha\left(t_{p}-t_{0}\right)\right)\right)
$$

where $w_{0}$ corresponds to a small probability for a platelet to be activated before reaching the growing thrombus and the quantities $\alpha$ and $t_{0}$ are defined by the equations:

$$
t_{0}=\frac{t_{\min }+t_{\max }}{2}, \alpha=\frac{2}{t_{\max }-t_{\min }} .
$$

The function $w\left(t_{p}\right)$ turns to be a step function when $t_{\max }=t_{\min }$.

The rate of change $d N / d t$ of the number of platelets attached to the growing thrombus can be given by the equation:

$$
\frac{d N}{d t}=n \pi R b v(R) w\left(t_{p}(R)\right) .
$$

where $n$ is the platelet concentration in the blood. With a help of the Eq. (2) the rate of change $d N / d t$ can be presented in the form:

$$
\frac{d N}{d t}=C_{4} n R b w \frac{Q b}{\left(R_{0}-R+b\right)\left(R_{0}^{2}-R^{2}\right)} .
$$

The flow rate $Q$ in (6) depends on the thrombus size. This dependence can be determined in the following way. We assume blood to be an incompressible fluid with constant viscosity and consider a streamline blood flow without turbulence in a tube with a radius $R_{0}$ and a length $L$. According to J.L.M. Poiseuille and G.G. Stokes, the dependence of blood velocity on the distance $r$ from the axis of the tube is given by the equation [12]:

$$
v(r)=\frac{\Delta P}{4 \eta L}\left(R_{0}^{2}-r^{2}\right)
$$


where $\eta \mathrm{a}$ is blood viscosity and $\Delta P$ is the pressure difference between the ends of the tube. Thus, for the blood flow rate $Q$ we get

$$
Q=\frac{\pi R_{0}^{4} \Delta P}{8 \eta L}
$$

Now the Eq. (7) can be rewritten as

$$
v(r)=\frac{2 Q\left(R_{0}^{2}-r^{2}\right)}{\pi R_{0}^{4}} .
$$

Consequently for the dissipated in the tube power due to viscosity $W_{D}$ we get the equation

$$
W_{D}=2 \pi \int_{0}^{R_{0}} \Delta P v(r) r d r=Q \Delta P,
$$

which with the help of Eq. (8) can be presented in the form

$$
W_{D}=\frac{8 \eta L}{\pi R_{0}^{4}} Q^{2}
$$

It is convenient to express Eq. (11) in terms of the area of the cross section $S_{0}$ of the tube:

$$
W_{D}=\frac{8 \pi \eta L}{S_{0}^{2}} Q^{2} .
$$

The generalization of Eq.(12) to the case of noncircular form of blood vessel results in the same expression (12) with the only change of $8 \pi$ for the dimensionless constant of the same order of magnitude:

$$
W_{D}=\frac{C_{6} \eta L}{S_{0}^{2}} Q^{2}
$$

To apply this result to the case under consideration we accept the following model for a blood vessel with a growing thrombus inside (Fig.5).

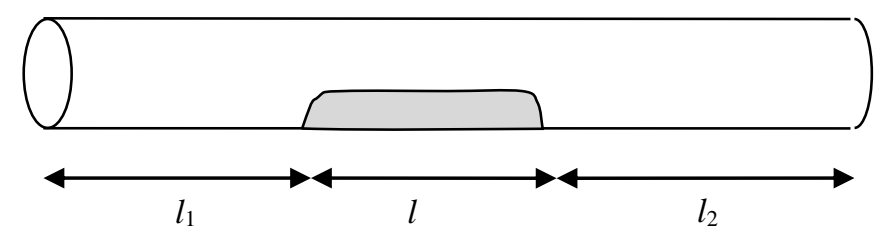

Fig.5

The calculation of the dissipated power in each part of the vessel gives the following result for the total dissipated power: 


$$
W_{D}=\eta\left(\frac{C_{6} l_{0}}{S_{0}^{2}}+\frac{C_{7} l}{\left(S_{0}-S\right)^{2}}\right) Q^{2}
$$

where $S_{0}-S$ is an area of the vessel cross-section at the point of thrombus location and $l_{0}=l_{1}+l_{2}$ is a total length of the vessel part without a thrombus.

The continuity equation is valid for the stationary viscous flow [12], so the power of external forces providing the movement of blood $W_{P}$ can be given by the following expression

$$
W_{p}=\Delta P Q .
$$

The power $W_{P}$ is equal to the dissipated power $W_{D}$. This condition leads to the following equation for the blood flow rate $Q$ :

$$
Q=\frac{\Delta P}{\eta\left(\frac{C_{6} l_{0}}{S_{0}^{2}}+\frac{C_{7} l}{\left(S_{0}-S\right)^{2}}\right)} .
$$

The set of Eqs. (3), (4), (6), and (16) is a closed one, if we take into account the relation between a number $N$ of platelets in a thrombus and its volume $\mathrm{Sl}$ :

$$
N=n_{t} S l
$$

where $n_{t}$ is a platelet concentration in a thrombus.

\section{Introduction of the dimensionless parameters}

Eq. (16) can be written in a different form if we introduce a dimensionless parameter $s$ equal to the ratio of the areas of cross sections of thrombus and blood vessel $s=S / S_{0}$ and take into account that the thrombus's length $l$ is proportional to its radius $R$ :

$$
Q=\frac{C_{8} \Delta P S_{0}^{2}}{\eta l_{0}\left(1+C_{9} \frac{\sqrt{S_{0}}}{l_{0}} \frac{\sqrt{s}}{(1-s)^{2}}\right)} .
$$

Introducing the dimensionless constant $k=C_{9} \sqrt{S_{0}} / l_{0}<<1$ we can write Eq. (18) in the following way:

$$
\frac{Q}{S_{0}}=\frac{C_{8} \Delta P S_{0}}{\eta l_{0}} \frac{1}{1+\frac{k \sqrt{s}}{(1-s)^{2}}} .
$$


The constant $\frac{C_{8} \Delta P S_{0}}{\eta l_{0}}$ has the dimension of velocity and can be interpreted as a mean value of blood flow velocity $v_{0}$ in the absence of thrombus. Then we get the following equation for $Q$ :

$$
Q=\frac{v_{0} S_{0}}{1+\frac{k \sqrt{s}}{(1-s)^{2}}}
$$

Eq. (3) for the time $t$ during which a platelet moves near the growing thrombus with the help of introduced constants $k, s$ and $v_{0}$ can be brought to the following form:

$$
t_{p}=C_{3} \frac{\sqrt{S_{0}}}{v_{0} \delta} \sqrt{s}(1-\sqrt{s}+\delta)(1-s)\left(1+\frac{k \sqrt{s}}{(1-s)^{2}}\right),
$$

where $\delta=b / \sqrt{S_{0}}$. The constant factor $T_{0}=C_{3} \sqrt{S_{0}} /\left(v_{0} \delta\right)$ in Eq. (21) has the dimension of time. The quantity $T_{0}$ is larger than the time a platelet needs to cover the distance $R_{0}$. The ratio of these quantities is of the order $R_{0} / b$. Qualitative estimations can be made on the basis of data presented in [1]: $R_{0}=30 \mathrm{mkm}, v_{0}=3$ $\mathrm{mm} / \mathrm{s}$. Assuming $C_{3}=1$ we get $T_{0}=.15 \mathrm{~s}$ what corresponds to the platelet activation time.

It is convenient to introduce the dimensionless time $\tau=t / T_{0}$. The equation for this quantity is obtained with a help of (21):

$$
\tau_{p}=\sqrt{s}(1-\sqrt{s}+\delta)(1-s)\left(1+\frac{k \sqrt{s}}{(1-s)^{2}}\right) \text {. }
$$

The dimensionless quantities $\tau_{\min }=t_{\min } / T_{0}, \tau_{\max }=t_{\max } / T_{0}$ и $\delta$ appear to be the parameters of the phenomenological theory of laser-induced thrombus formation. The quantities $t_{p}, t_{\min }$ and $t_{\max }$ in the expression (4) for the probability $w(t)$ should be changed for $\tau_{p}, \tau_{\min }$ и $\tau_{\max }$. Eq. (6) for the rate of change of thrombus size in these new terms gets the form:

$$
\frac{d N}{d t}=C_{10} n v_{0} S_{0} \delta^{2} \frac{w\left(\tau_{p}(s)\right) \sqrt{s}}{(1-\sqrt{s}+\delta)(1-s)\left(1+\frac{k \sqrt{s}}{(1-s)^{2}}\right)}
$$

Eq. (17) written in the terms of the dimensionless parameter $s$ and $\tau$

$$
N=C_{11} n_{t} S_{0}^{3 / 2} s^{3 / 2}
$$


permits to transform Eq. (23) for $d N / d t$ into the equation for $d s / d \tau$. Finally, we get the following closed set of equations for the phenomenological model of laserinduced thrombus formation in vivo:

$$
\begin{gathered}
\frac{d s}{d \tau}=C \frac{n}{n_{t}} \frac{w\left(\tau_{p}(s)\right)}{(1-\sqrt{s}+\delta)(1-s)\left(1+\frac{k \sqrt{s}}{(1-s)^{2}}\right)}, \\
w\left(\tau_{p}\right)=w_{0}+\frac{1}{2}\left(1+w_{0}+\left(1-w_{0}\right) \operatorname{th}\left(\alpha\left(\tau_{p}-\tau_{0}\right)\right)\right), \\
\tau_{0}=\frac{\tau_{\min }+\tau_{\max }}{2}, \alpha=\frac{2}{\tau_{\max }-\tau_{\min }}, \\
\tau_{p}=\sqrt{s}(1-\sqrt{s}+\delta)(1-s)\left(1+\frac{k \sqrt{s}}{(1-s)^{2}}\right) .
\end{gathered}
$$

Comparison with the experimental data requires the knowledge of a mean value of blood velocity $\langle v\rangle=\frac{Q}{S_{0}-S}$ in the vicinity of a thrombus. In terms of the introduced dimensionless parameters it can be written in the form:

$$
\langle v\rangle=\frac{v_{0}}{1-s+\frac{k \sqrt{s}}{1-s}} .
$$

It follows from Eq. (26) that $\langle v\rangle$ is increasing like $(1-s)^{-1}$, when dissipation is small, and tends to zero when $\mathrm{s} \rightarrow 1$.

The described model contains one parameter $T_{0}$ with the dimension of time, one parameter $v_{0}$ with the dimension of velocity, two parameters $n$ and $n_{t}$ with the dimension of concentration, and the dimensionless parameters $C, \delta, w_{0}, k, \tau_{\min }$, $\tau_{\max }$. The product $T_{0} \delta$ corresponds to the time a platelet needs to cover a distance $l$ equal to the thrombus length. It then follows from the equations of the model that the ratio of the time of thrombus growth to $T_{0}$ is of the order $n_{t} / n$. If we assume the time of thrombus growth to be equal $200 \mathrm{~s}$, and $T_{0}=.15 \mathrm{~s}$, we get $n_{t} / n=1300$. The concentration of platelets in blood is of the order $n=(150 \div 400) \cdot 10^{3} \mathrm{~mm}^{-3}$ and their concentration in blood can be roughly estimated as $n_{t} \approx b^{-3}=0.1 \div 1 \mathrm{mkm}^{-3}$. It gives for $n_{t} / n$ the value equal to $250 \div 7000$, what is in a reasonable correspondence with the value presented above.

The results of numerical calculations on the basis of the equations (25) are shown on Fig.6 $(v(t))$ and Fig.7 $(s(t)$ and $v(t))$ for the following values of the dimensionless parameters: $k=0,05 . \delta=0.05, w_{0}=0.1, \tau_{\min }=0.17, \tau_{\max }=0.22$. The con- 
stants $v_{0}$ and $C n / n_{t}$ were put equal to 1 . The comparison with the experimental data on Fig. 1 and Fig. 2 demands the coinciding scales what can be achieved for the quite reasonable vales $v_{0}=10 \mathrm{~mm} / \mathrm{s}$ and $C=1, n_{t} / n=300$.

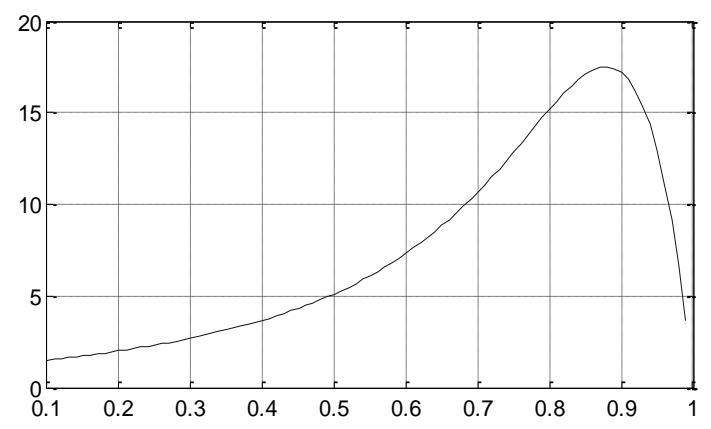

Fig.6.

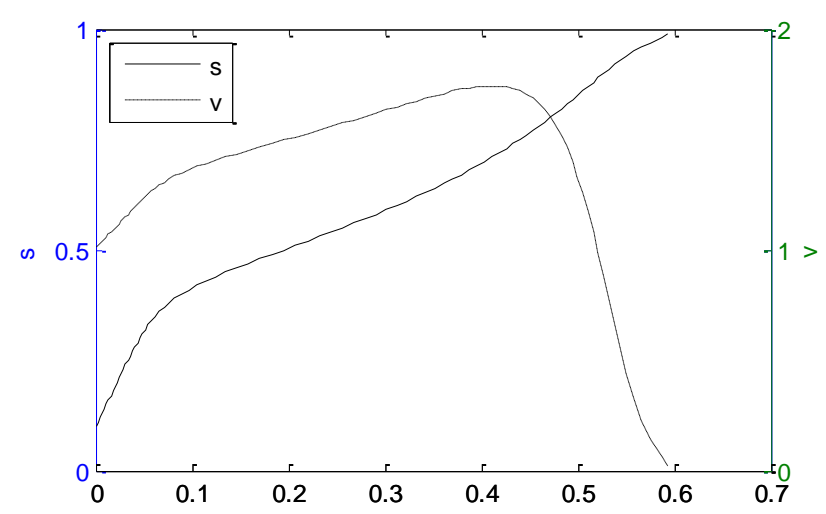

Fig. 7

\section{Discussion}

The comparison of the modeling curves in [1] and in this paper reveals their similar shape and qualitative agreement with experimental grow curves. It ought to be noted that the presented modeling curves are universal for microvessels, i.e. can simulate the thrombus growth either in arterioles or in venules in the same way as in [1]. The experimental grow cures presented in [21] for both types of microvessels have a plateau as a main specific feature and both models under consideration correspond to this circumstance. The analysis of experimental reports that describe the influence of different factors on thrombus formation confirms that the most important of them can be taken into account in the present theoretical framework as well as in [1]. The flexibility of the models enables one to extent both of them to the description of any scenario of experimental thrombosis. 
But the phenomenological models developed in [1] and in this paper differ essentially in the initial assumptions.

First of all, the assumption of a certain dependence of platelet's activation time on the distance from the damaged wall site revealed in [1] in explicit form is absent in the model in this paper. Secondly, the platelet concentration in the blood flow here is considered to be constant, but in [1] the experimentally confirmed realistic profile of platelet distribution in the blood flow served as a key stone of the model. Finally, the choice of the phenomenological function for the dependence of the blood flow velocity on the thrombus size made in [1] on the basis of the continuity equation and biological considerations is changed here for the explicit calculation of this dependence on the basis of viscous fluid dynamics with subsequent introduction of the probability function describing the processes of platelet attachment and detachment. It means that in the presented model this property gets its explanation in pure physics terms.

Both models under consideration are typical phenomenological theories of thrombus development that are based on fundamental reliable experimental results. Their phenomenological parameters are determined by the comparison with experimental data. Both models have the stochastic character, but they differ also in the way of construction. For example, Eq. (12) in [1] looks like dynamical equation which describes "slow" process in thrombus growth. Its stochastic character is hidden by the rough time scale and is revealed by an implicit averaging of "quick" processes over the "rough" time length $d t$. On the contrary the stochastic character of the present model is revealed in explicit way by the introduction of the probability function (4). But at the same time both models are based on Bogolubov's hierarchy of time scales and in this sense are linked genetically.

It is easy to see that the differences of the phenomenological models under consideration correspond to different experimental pictures of thrombus formation. The model developed in [1] corresponds to a case of a friable thrombus which is to some extent transparent for the moving platelets: platelets can penetrate inside the gradually growing thrombus and can be activated there. In this case the assumptions concerning the activation time and the platelet distribution in the blood flow are important. The model developed in this paper corresponds to a case of a solid thrombus which increasing in size shifts the border layer of blood and thus remains practically in the same conditions concerning platelet distribution and activation time. Both cases of thrombus formation are possible in experiment and the developed phenomenological models demonstrate the insensitivity of the resulting macroscopic picture on the microscopic details of the process. This insensitivity also testifies to the possibility of neglecting many of the described in [11] specific biological details of thrombus formation in a process of creating phenomenological models. 
From the mathematical point of view such insensitivity means that different biological factors that correspond to various mechanisms of interaction between the members of thrombus formation process in reality make identical or at least similar contributions to the resulting picture of the phenomenon. It also means that the phenomenological models under consideration are rather universal in the sense that they both do not take into account in explicit form many specific details, such as concentration of fibrinogen or the length of fibrinogen stands. Yet they both produce remarkably true-to-life results in the qualitative description of laser-induced thrombus formation and establish a certain direction of a possible development of microscopic models.

In conclusion, speaking of the meaning of such simple phenomenological models? We will quote Philip W. Anderson's Nobel prize address, presented in [3]: " The art of model-building is the exclusion of real but irrelevant parts of the problem and entails hazards for the builder and the reader. The builder may leave out something genuinely relevant and the reader, armed with too sophisticated an experimental probe, may take literally a schematized model. Very often such a simplified model throws more light on the real working of nature...".

\section{References}

[1] A. Alenitsyn, A. Kondratyev, I. Mikhailova, I. Siddique, Mathematical modeling of thrombus growth in mesenteric vessels, Mathematical Biosciences, 224 (2010) 29-34. http://dx.doi.org/10.1016/j.mbs.2009.12.005

[2] N. Begent, G.V.R. Born, Growth rate in vivo of platelet thrombi, produced by iontophoresis of ADP as a function of mean blood velocity, Nature, 227 (1970) 926-930. http://dx.doi.org/10.1038/227926a0

[3] K. B. Blagoev, K. Shukla, and H. Levine. We need theoretical physics approaches to study living systems, Phys. Biol., 10 (2013) 040201.

http://dx.doi.org/10.1088/1478-3975/10/4/040201

[4] A. Bonnefoy, Q. Lui, C. Legrand, M. Frojmovic, Efficiency of platelet adhesion to fibrinogen depends on both cell activation and flow, Biophys. J., 78 (2000) 2834-2843. http://dx.doi.org/10.1016/s0006-3495(00)76826-3

[5] T. David, P. G. Walker, Activation and extinction models for platelet adhesion, Biorheology, 39 (2002) 293-298.

[6] B. Furie, B.C. Furie, Thrombus formation in vivo, J. Clin. Invest., 115 (2005) 3355-3362. http://dx.doi.org/10.1172/jci26987 
[7] M. F. Hockin, K. J. Jones, S. J. Everse, K. G. Mann, A model for the stoichiometric regulation of blood coagulation, J. Bio. Chem., 277 (2002) 1832218333. http://dx.doi.org/10.1074/jbc.m201173200

[8] A. Kondratyev, I. Mikhailova, Mathematical Modeling of Laser-Induced Thrombus Formation in Microvasculature, in: Mathematical Modeling, Ed. Christopher R. Brennan. N.Y.: Nova Science Publishers, Inc., 2011. Ch. 5.

[9] A. S. Kondratyev, I. A. Mikhailova, N. N. Petrishchev, Effect of blood flow velocity on platelet thrombi formation in microvessels, Biophysics, 35 (1990) 469472.

[10] S. Kulharni, S. M. Dopheide, C. L. Yap, C. Ravanat et al, A revised model of platelet aggregation, J. Clin. Invest., 105 (2000) 783-791.

http://dx.doi.org/10.1172/jci7569

[11] A. L. Kuharsky, A. L. Fogelson, Surface-mediated control of blood coagulation: the role of binding site densities and platelet deposition, Biophys. J., 80 (2001) 1050-1074. http://dx.doi.org/10.1016/s0006-3495(01)76085-7

[12] L. D. Landau, E. M. Lifshitz. Fluid Mechanics. Vol. 6. Butterworth Heinemann, 1987. http://dx.doi.org/10.1016/b978-0-08-033933-7.50014-3

[13] S. Mordon, S. Begu, B. Buys, C. Tourne-Peteilh, J. Devoisselle, Study of platelet behavior in vivo after endothelial stimulation with laser irradiation using fluorescence intravital videomicroscopy and PEGylated liposome staining, Microvasc. Res., 64 (2002) 316-325. http://dx.doi.org/10.1006/mvre.2002.2435

[14] J. D. Murray, Mathematical Biology, N.Y.: Springer-Verlag, 2004. 551 p.

[15] R. Ouared, B. Chopard, B. Stahl, D. A. Rufenacht, H. Yilmaz, G. Courbebaisse, Thrombosis modeling in intracranial aneurysms: a lattice Boltzmann numerical algorithm, Comput. Phys. Commun., 179 (2008) 128-131. http://dx.doi.org/10.1016/j.cpc.2008.01.021

[16] N. N. Petrishchev, A. S. Kondratyev, I. A. Mikhailova, Effect of blood flow on thrombus growth in mesenteric vessels, $6^{\text {th }}$ World Congress for Microcirculation, Munich (Germany) (1996) 487-490.

[17] I. V. Pivkin, P. D. Richardson, G. Karniadakis, Blood flow velocity effects and role of activation delay time on growth and form of platelet thrombi, Proc. Natl. Acad. Sci. USA, 103 (2006) 17164-17169.

http://dx.doi.org/10.1073/pnas.0608546103 
[18] P. D. Richardson, Effect of blood flow velocity on growth rate of platelet thrombi, Nature, 245 (1973) 103-104. http://dx.doi.org/10.1038/245103a0

[19] Z. M. Ruggeri, Old concepts and new developments in the study of platelet aggregation, J. Clin. Invest. 105 (2000) 699-701.

http://dx.doi.org/10.1172/jci9604

[20] Z. M. Ruggeri, G. L. Mendolicchio, Adhesion mechanisms in platelet function, Circ. Res., 100 (2007) 1673-1685.

http://dx.doi.org/10.1161/01.res.0000267878.97021.ab

[21] M. Sato, N. Ohshima, Hemodynamics at stenosis formed by growing platelet thrombi in mesenteric microvasculature of rat, Microvasc. Res., 31 (1986) 66-76. http://dx.doi.org/10.1016/0026-2862(86)90007-5

[22] T. W. Secomb, Theoretical Models for Regulation of Blood Flow, Microcirculation, 15 (2008) 765-775.

http://dx.doi.org/10.1080/10739680802350112

[23] A. Tailor, D. Cooper, D. N. Granger, Platelet-Vessel Wall Interactions in the Microcirculation, 12 (2005) 275-285.

http://dx.doi.org/10.1080/10739680590925691

[24] A. A. Tokarev, A. A. Butylin, F. I. Ataullakhanov, Platelet Adhesion from Shear Blood Flow is Controlled by Near-Wall Rebounding Collisions with Erythrocytes, Biophys. J., 100 (2011) 799-808.

http://dx.doi.org/10.1016/j.bpj.2010.12.3740

[25] A. A. Tokarev, Yu. V. Krasotkina, M. V. Ovanesov, M. A. Panteleev, M. A. Azhigirova, V. A. Volpert, F. I. Ataullakhanov, A. A. Butylin, Spatial Dynamics of Contact-Activated Fibrin Clot Formation in vitro and in silico in Haemophilia B: Effects of Severity and Ahemphil B Treatment, Mathematical modeling of natural phenomena, 1 (2006) 124-137. http://dx.doi.org/10.1051/mmnp:2008007

[26] A. Tokarev, I. Sirakov, G. Panasenko, V. Volpert,E. Shnjl, A. Butylin, F. Ataullakhanov, Continuous Mathematical Model of Platelet Thrombus Formation in Blood Flow, Russian Journal of Numerical Analysis and Mathematical Modeling, 27 (2012) 191-212. http://dx.doi.org/10.1515/rnam-2012-0011

[27] D. Varga-Szabo, I. Pleines, B. Nieswandt, Cell adhesion mechanisms in platelets, Arterioscler. Thromb. Vasc. Biol., 28 (2008) 403-412.

http://dx.doi.org/10.1161/atvbaha.107.150474 
[28] D. Wootton, C. Marcou, S. Hanson, D. Ku, A mechanistic model of acute platelet accumulation in thrombogenic stenoses, Ann. Biom. Eng., 29 (2001) 321329. http://dx.doi.org/10.1114/1.1359449

[29] Z. Xu, N. Chen, M. M. Kamocka, E. D. Rosen, M. S. Alber, Multiscale model of thrombus development, J. Royal Soc. Interface, 5 (2008) 705-722.

http://dx.doi.org/10.1098/rsif.2007.1202

[30] Z. Xu, N. Chen, S. Shadden, J. E. Marsden, M. M. Kamocka, E. D. Rosen, M. S. Alber, Study of blood flow impact on growth of thrombi using a multiscale model, Soft Matter, 5 (2009) 769-779.

http://dx.doi.org/10.1039/b812429a

Received: September 17, 2014; Published: January 7, 2015 